\title{
Entropic force of polymers on a cone tip
}

\author{
Mohammad F. Maghrebi,, , * Yacov Kantor, ${ }^{2}$ and Mehran Kardar ${ }^{1}$ \\ ${ }^{1}$ Massachusetts Institute of Technology, Department of Physics, Cambridge, Massachusetts 02139, USA \\ ${ }^{2}$ Raymond and Beverly Sackler School of Physics and Astronomy, Tel Aviv University, Tel Aviv 69978, Israel
}

(Dated: June 11, 2018)

\begin{abstract}
We consider polymers attached to the tip of a cone, and the resulting force due to entropy loss on approaching a plate (or another cone). At separations shorter than the polymer radius of gyration $R_{g}$, the only relevant length scale is the tip-plate (or tip-tip) separation $h$, and the entropic force is given by $F=\mathcal{A} k_{B} T / h$. The universal amplitude $\mathcal{A}$ can be related to (geometry dependent) correlation exponents of long polymers. We compute $\mathcal{A}$ for phantom polymers, and for self-avoiding (including star) polymers by $\epsilon$-expansion, as well as by numerical simulations in 3 dimensions.
\end{abstract}

PACS numbers: 64.60.F- 82.35.Lr 05.40.Fb

Single molecule manipulation [1 5] using techniques such as atomic force microscopy (AFM) [6] , microneedles [7], optical [8, 9] and magnetic [10] tweezers enable extremely detailed study of geometry and forces in long polymers. The positional accuracy of AFM tip [5, 11] can be as good as few nm, while the forces of order of $1 \mathrm{pN}$ can be measured, and measurements can be carried out in almost biological conditions [12, 13]. These enhanced sensitivities bring us to the range where entropic forces of long polymers in a solvent can be significant even when the deformation of the polymer is relatively slight. While the main thrust of the experimental research is extraction of specific information from the force-displacement behaviors of the polymers, certain features are independent of the microscopic details [14], but depend on the probe shape, as discussed in this work.

We consider an idealized set-up in which a polymer is attached to the tip of a solid cone. The cone approaching a plate (or another cone) exemplifies a geometry in which the only (non-microscopic) length scale is provided by the tip-plate (or tip-tip) separation $h$. Fluctuating polymers typify self-similar variations at scales intermediate between microscopic (persistence length $a$ ) and macroscopic. The latter is set by the radius of gyration which grows with the number of monomers through the scaling relation $R_{g} \propto N^{\nu}$. Thus when a cone-tip-attached polymer approaches a plate, at separations $a \ll h \ll R_{g}$ the only relevant length scale is $h$, and on dimensional grounds, the force due to loss of entropy must behave as

$$
F=\mathcal{A} \frac{k_{B} T}{h} .
$$

Such a force law should apply to all circumstances where the separation provides the only relevant length scale. The amplitude $\mathcal{A}$ will depend on geometric factors such as the opening angle of the cone $\Theta$ (and if tilted, on the corresponding angle). One could presume, that the dimensionless amplitude may also depend on microscopic

\footnotetext{
* Email: magrebi@mit.edu
}

properties on the polymer. However, in case of cone-tippolymers we shall demonstrate that the amplitude $\mathcal{A}$ can be related to universal (and shape dependent) polymer exponents. The simple force law of Eq. (11) follows easily from various polymer scaling forms (see, e.g. the derivation below) such as in Refs. [15 17], and should be part of polymer lore. Surprisingly, we could not find an explicit reference to it in any of the standard polymer textbooks.

A polymer attached to the tip of an AFM is approximated as linked to the apex of a cone as depicted in Fig. 11. With the cone far away from a plate $\left(h \gg R_{g}\right)$, the number of configurations of the polymer grows with the number of monomers as

$$
\mathcal{N}_{\mathrm{c}}=b z^{N} N^{\gamma_{\mathrm{c}}(\Theta)-1},
$$

where the effective coordination number $z$, as well as the pre-factor $b$, depend on the microscopic details, while the 'universal' exponent $\gamma_{\mathrm{c}}$ only depends on the cone angle. When the cone touches the plate as in Fig. 1, the number of configurations is reduced to $\mathcal{N}_{\text {cp }}$ with the same form as Eq. (2), but with a different exponent $\gamma_{\mathrm{cp}}(\Theta)$. We shall henceforth use the exponent subscript 's' (as in $\gamma_{\mathrm{s}}$ ) to refer to the above cases, with " $\mathrm{s}=\mathrm{c}$ " for cone and "s=cp" for cone+plate; the absence of a subscript (as in $\gamma$ ) will signify a free polymer. The work done against the entropic force in bringing in the tip from afar to contact the plate can now be computed from Eq. (1) as

$$
W=\int_{a}^{R_{g}} \mathrm{~d} h \mathcal{A} \frac{k_{B} T}{h}=\mathcal{A} k_{B} T \ln \frac{R_{g}}{a}=\mathcal{A} \nu k_{B} T \ln N .
$$

The work can also be computed from the change in free energies between the final and initial states, due to the change in entropy, as

$$
\Delta \mathcal{F}=-T \Delta \mathcal{S}=T \mathcal{S}_{\mathrm{c}}-T \mathcal{S}_{\mathrm{cp}}=k_{B} T\left(\gamma_{\mathrm{c}}-\gamma_{\mathrm{cp}}\right) \ln N,
$$

where the entropy $\mathcal{S}=-k_{B} \ln \mathcal{N}$ was computed from Eq. (2). By equating $W$ and $\Delta \mathcal{F}$ we find

$$
\mathcal{A}=\frac{\gamma_{\mathrm{c}}-\gamma_{\mathrm{cp}}}{\nu}=\eta_{\mathrm{cp}}-\eta_{\mathrm{c}} ;
$$

the final result obtained from the scaling law $\gamma_{\mathrm{s}}=$ $\left(2-\eta_{\mathrm{s}}\right) \nu$, where $\eta$ characterizes the anomalous decay of correlations $\left(\sim 1 / r^{d-2+\eta}\right)$. 


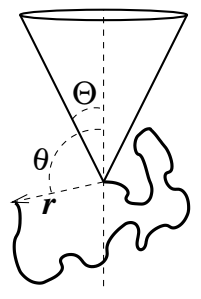

(a)

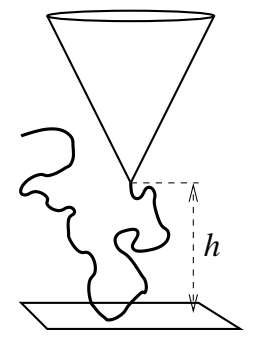

(b)

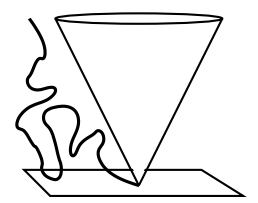

(c)
FIG. 1. (a) Polymer attached to the tip of a solid cone with apex semi-angle $\Theta$ (configuration "c"); positions are described by the spherical coordinates $r, \theta$ and azimuthal angle $\phi$ (not shown). (b) The tip is at a distance $h \ll R_{g}$ from the plate. (c) The tip touching the plate (configuration "cp").

In the above discussion we have assumed that the only interaction between the polymer and the surfaces is due to hard-core exclusion. Attractive interactions between the polymer and surface will introduce temperature dependent corrections, and an additional size scale. Weak interactions are asymptotically irrelevant, but strong interactions may lead to a phase in which the polymer is absorbed to the surface, where the entropic considerations presented here will no longer be appropriate.

We have derived Eq. (4) for the specific case of a cone and a plate. However, this equation can be applied to any situation where in the limits of large and vanishing separations we have scale-free shapes. In particular, the same rule can be applied when a linear or star polymer is brought from infinity to a contact with a repulsive plane, since both extremes have no length-scale, leading to exact expressions for the force constant [16]. However, if the AFM tip is slightly rounded, an additional length scale is introduced, and one may expect a non-trivial crossover between various regimes [18].

We have thus reduced the computation of the force to calculation of correlation functions. In the absence of self-avoidance and other interactions, correlations of the so-called ideal or phantom polymer (henceforth denoted by subscript 0 ) are the same as a free-field theory, satisfying (at scales shorter than $R_{g}$ ) the Laplace equation $\nabla^{2} G_{0}\left(\mathbf{r}, \mathbf{r}^{\prime}\right)=-\delta\left(\mathbf{r}-\mathbf{r}^{\prime}\right)$ [14]. With one point at a short distance $a$ from the cone, correlations behave as $G_{0}(a, \mathbf{r}) \sim a^{\eta_{0}} / r^{d-2+\eta_{0}} \Psi(\theta)$, where $r$ and $\theta$ denote the distance of $\mathbf{r}$ from the tip, and the angle of $\mathbf{r}$ to the cone axis. The change in scaling from a free phantom polymer is captured by $(a / r)^{\eta_{0}}$, and $\Psi(\theta)$ is a dimensionless function depending only on the polar angle due to the symmetry of the geometry. (We consider a generalized cone in $d$ spatial dimensions characterized by a single polar angle $\theta$, and $d-2$ azimuthal angles $\phi, \psi, \cdots$.) Substituting the above form in the Laplace equation, we find that the exponent $\eta_{0}$ satisfies

$$
\frac{1}{(\sin \theta)^{d-2}} \frac{d}{d \theta}\left[(\sin \theta)^{d-2} \frac{d \Psi}{d \theta}\right]+\eta_{0}\left(d-2+\eta_{0}\right) \Psi(\theta)=0,
$$

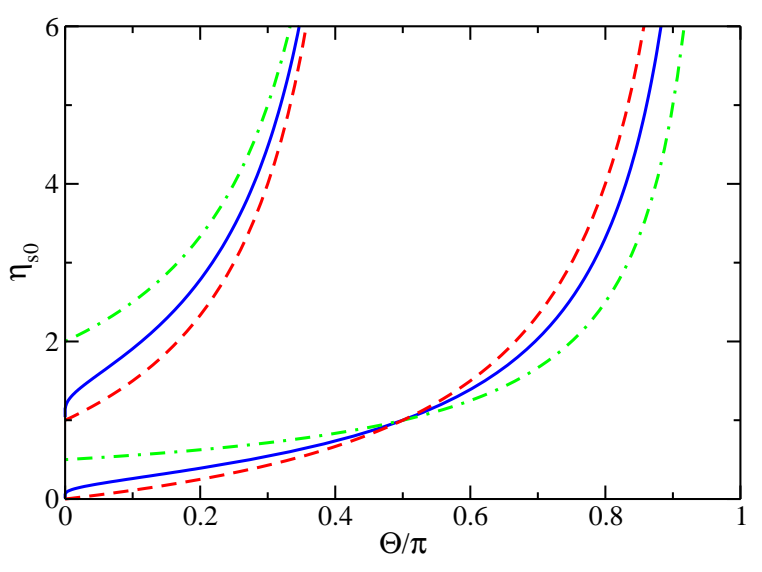

FIG. 2. (Color online) The exponent $\eta_{\mathrm{s} 0}$ for ideal polymers in $d=2$ (dot-dashed), 3 (solid), 4 (dashed) for cone ("s=c") of angle $\Theta$ (bottom curves), and "s=cp" (top curves).

with an appropriate boundary condition on $\Psi$. For an isolated cone, the function $\Psi$ must be positive and regular outside the cone, with $d \Psi /\left.d \theta\right|_{\theta=\pi}=0$ to avoid a cusp on the symmetry axis, and $\Psi(\Theta)=0$ on the cone surface. For the cone+plate, the appropriate solution is positive and vanishes both at $\theta=\Theta$ and $\theta=\pi / 2$. The first case was considered by Ben-Naim and Krapivsky [19] in connection with diffusion near an absorbing boundary [20], and we follow these derivations. The solution in general $d$ requires the use of associated Legendre functions, but simplifies in a few cases described below.

- For $d=2$, Eq. (6) reduces to $\Psi^{\prime \prime}+\eta_{0}^{2} \Psi=0$; solved by linear combinations of $\sin \left(\eta_{0} \theta\right)$ and $\cos \left(\eta_{0} \theta\right)$ to yield

$$
\eta_{\mathrm{c} 0}=\frac{\pi}{2(\pi-\Theta)}, \quad \text { and } \quad \eta_{\mathrm{cp} 0}=\frac{2 \pi}{\pi-2 \Theta} .
$$

Both results (depicted in Fig. 2) go to a finite value as $\Theta \rightarrow 0$, reflecting the strong reduction in configurations due to the remnant (barrier) line, and $\eta_{0} \rightarrow \infty$ when the boundaries confine the polymer to a vanishing sector.

- For $d=4$, the substitution $\Psi=u / \sin \theta$ simplifies Eq. (6) to $u^{\prime \prime}+\left(\eta_{0}+1\right)^{2} u=0$, solved by a linear combination of $\sin \left[\left(\eta_{0}+1\right) \theta\right]$ and $\cos \left[\left(\eta_{0}+1\right) \theta\right]$, and we find

$$
\eta_{\mathrm{c} 0}=\frac{\Theta}{\pi-\Theta}, \quad \text { and } \quad \eta_{\mathrm{cp} 0}=\frac{\pi+2 \Theta}{\pi-2 \Theta}
$$

depicted by the bottom and top dashed lines in Fig. 2. The cone exponent $\eta_{\mathrm{c} 0}$ vanishes linearly with $\Theta$-a needle in four dimensions is invisible.

- For $d=3$, Eq. (6) is solved by a linear combination of regular Legendre functions $P_{\eta_{0}}(\cos \theta)$ and $Q_{\eta_{0}}(\cos \theta)$. The resulting exponents (which cannot be cast as simple functions), are plotted as solid lines in Fig. 2, The exponent $\eta_{\mathrm{c} 0}(\Theta)$ vanishes with the angle, but both curves approach their limiting value as $\Theta \rightarrow 0$ with infinite slope via a logarithmic singularity $(\sim 1 /|\ln \Theta|)$. 
- For all $d$, the cone becomes a plate for $\Theta=\pi / 2$. Correlations with one point approaching a surface are easily obtained by the method of images [21] leading to $\eta_{\mathrm{c} 0}=1$, which is clearly seen in Fig. 2, For $3<d<4$, both exponents approach their limiting value when $\Theta \rightarrow 0$ as $\Theta^{p_{0}}$ with $p_{0}=d-3$ :

$$
\begin{aligned}
\eta_{\mathrm{c} 0} & =\frac{\Gamma(1-\epsilon / 2)}{\sqrt{\pi} \Gamma(1 / 2-\epsilon / 2)} \Theta^{1-\epsilon}, \quad \text { and } \\
\eta_{\mathrm{cp} 0} & =1+\frac{4 \Gamma(2-\epsilon / 2)}{\sqrt{\pi} \Gamma(1 / 2-\epsilon / 2)} \Theta^{1-\epsilon},
\end{aligned}
$$

where $d=4-\epsilon$. These equations reflect the fact that the two dimensional phantom polymer will not intersect the remnant line in $d>3$. For $d<3$, the limiting value is different from the case without any cone indicating the finite probability of intersection of the polymer with the line.

Universal aspects of swollen (coil) polymers with shortrange interactions can be modeled by a self-avoiding walk (SAW). In $d=3$, a SAW in empty space has exponent $\gamma \approx 1.158$ [22], while in $d=2, \gamma=43 / 32$ [23] (for ideal polymers $\gamma=1$ at any $d$ ). There are a number of results regarding $\gamma_{\mathrm{s}}$ for polymers confined by wedges in 2D and 3D [24-29]: A SAW anchored at the origin and confined to a solid wedge (in 3D) or a planar wedge (in 2D) has an angle-dependent $\gamma_{\mathrm{s}}$ that diverges as the confining angle vanishes. Studies of a polymer attached to the tip of a $2 \mathrm{D}$ sector in $3 \mathrm{D}$, and to the apex of a cone have been performed [30]. Extensive analytical [31, 32] and numerical [28, 29] studies of SAWs anchored to a solid plate in 3D find $\gamma_{\mathrm{s}} \equiv \gamma_{1} \approx 0.70$ [28], or 0.68 [33]. For ideal polymers $\gamma_{1}=1 / 2$ for any $d$. We are unaware of specific results for the geometries depicted in Fig. 1, and report below our numerical and analytical estimates.

We performed numerical simulations for SAWs of lengths $N=16,32, \ldots, 1024$ on a cubic lattice. The SAWs were generated by a dimerization method [34, 35] in which an unbiased $N$-step SAW is created by attempting to join two $N / 2$-step SAWs previously obtained by the same algorithm. We generated $10^{8} \mathrm{SAWs}$ for each $N$, each of which is attached to the origin and checked whether it touches a cone (or cone+plate). The probability that an $N$-step SAW does not intersect the excluded space is the ratio of permitted number of walks to the total number of SAWs, i.e. $p_{N}=\mathcal{N}_{\mathrm{s}} / \mathcal{N} \sim N^{\gamma_{\mathrm{s}}-1} / N^{\gamma-1}=$ $N^{\Delta \gamma_{\mathrm{s}}}$. The ratio $p_{N} / p_{2 N}=2^{\Delta \gamma_{\mathrm{s}}}$ is then used to estimate $\Delta \gamma_{\mathrm{s}}$ for each sequential pair of $N \mathrm{~s}$. The results are extrapolated to $N \rightarrow \infty$ by plotting the estimates versus $1 / \sqrt{N}$; errors are due to both finite sample size and insufficiently large $N$. The estimated exponents (full symbols in Fig. [3) are rather close to the results for ideal polymers. This apparent proximity is somewhat misleading as $\Delta \gamma_{\mathrm{s}}=\gamma-\gamma_{\mathrm{s}}$ masks an appreciable shift in both $\gamma$ and $\gamma_{\mathrm{s}}$. For example, for a SAW near an isolated cone we expect $\Delta \gamma(\Theta=\pi / 2)=\gamma-\gamma_{1}=0.44-0.48$, very close to ideal polymer result of $1 / 2$; our numerical estimate at $\Theta=\pi / 2$ is $0.477 \pm 0.004$.

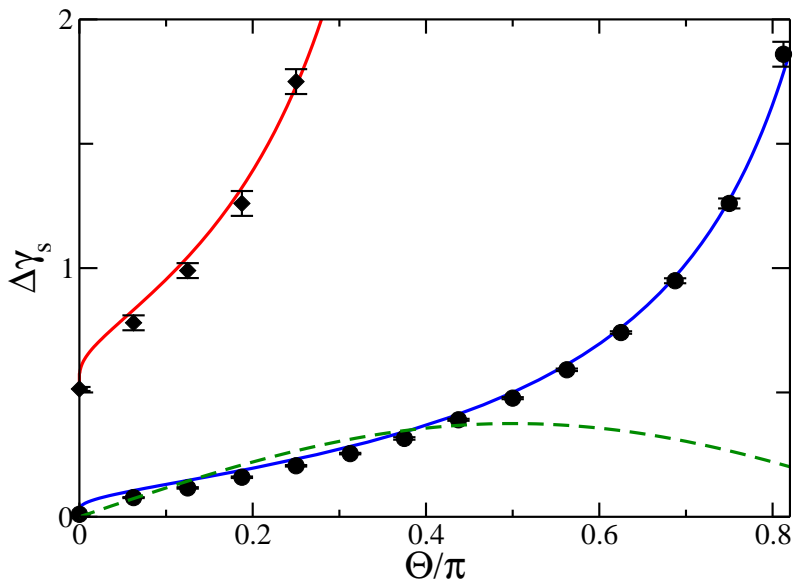

FIG. 3. (Color online) Dependence of the exponent difference $\Delta \gamma_{s}=\gamma-\gamma_{\mathrm{s}}$ on angle $\Theta$ for the cone+plate (top curves), and an isolated cone (bottom curves). The solid curves are the results for an ideal polymer, while the data points represent numerical results for SAWs in the same geometry. All error bars are estimates of the $N \rightarrow \infty$ extrapolation error. The dashed line depicts prediction of the $\epsilon$-expansion in Ref. [30].

The dashed line in Fig. 33 depicts the result [30] of an $\epsilon=(4-d)$ expansion in which the 2-dimensional surface of the cone is treated as a weakly repulsive potential. The lowest order result, $\Delta \gamma_{\mathrm{c}}=(3 \epsilon / 8) \sin \Theta$, captures some features of the numerics for $\Theta<\pi / 2$, but fails dismally for $\Theta>\pi / 2$ (since the polymer simply jumps to the larger space inside the cone). It also fails to capture the correct behavior as $\Theta \rightarrow 0$. A better approach is to exclude the interior of the cone, and to this end we follow the work of Cardy 25, 26] for the wedge geometry. The corresponding computations for a cone are more complicated, and we relied upon recent results on the electrodynamic Casimir force on a cone [36].

For an $\epsilon$-expansion we need the full non-interacting Green's function in 4-dimensions, going beyond the large separation asymptotic form obtained via Eq. (6). (For a general $\epsilon$-expansion, one has to know the Green's function in $4-\epsilon$ dimensions, however, we carry out this expansion only to first order in $\epsilon$. Since the strength of the interaction is linear in $\epsilon$, it suffices to obtain the Green's function in 4-dimensions.) Following Ref. [36], this is given by

$$
\begin{gathered}
G_{0}\left(x, x^{\prime}\right)=\sum_{k l m} \frac{(-1)^{l} \pi}{2} \frac{\Gamma\left(\rho_{k}+l+1\right)}{\sin \left(\rho_{k} \pi\right) \Gamma\left(\rho_{k}-l\right)} \frac{P_{\rho_{k}-1 / 2}^{-l-1 / 2}(\cos \Theta)}{\partial_{\rho_{k}} P_{\rho_{k}-1 / 2}^{-l-1 / 2}(-\cos \Theta)} \\
\frac{r_{<}^{\rho_{k}-1}}{r_{>}^{\rho_{k}+1}} \frac{P_{\rho_{k}-1 / 2}^{-l-1 / 2}(-\cos \theta) Y_{l m}(\psi, \phi)}{\sqrt{\sin \theta}} \frac{P_{\rho_{k}-1 / 2}^{-l-1 / 2}\left(-\cos \theta^{\prime}\right) Y_{l m}^{\star}\left(\psi^{\prime}, \phi^{\prime}\right)}{\sqrt{\sin \theta^{\prime}}},(10)
\end{gathered}
$$

where the sum is over the triplet of integers $k>0, l \geq 0$, and $-l \leq m \leq+l$. The important exponent $\rho_{k}$ is the $k$-th root of the transcendental equation $P_{\rho_{k}-1 / 2}^{-l-1 / 2}(-\cos \Theta)=$ 0 . This Green's function is broken up in radii $(r<$ and 
$r^{>}$), as appropriate to a polymer in the presence of a cone with one endpoint close to the tip and the other end far away.

The interacting Green's function at first order is obtained by subtracting polymer configurations that selfintersect, forming an intermediate loop, as

$$
G_{1}=G_{0}-u \int \mathrm{d}^{4} x^{\prime \prime} G_{0}\left(x, x^{\prime \prime}\right) G_{0}^{r}\left(x^{\prime \prime}, x^{\prime \prime}\right) G_{0}\left(x^{\prime \prime}, x^{\prime}\right) .
$$

In the language of quantum field theory, the first term is the "free" Green's function, albeit in the presence of external boundary conditions, while the second term is the one-loop correction. Note that the intermediate Green's function is regularized by subtracting the Green's function in empty space, hence the superscript $r$. In the above equation, $u$ is the strength of the self-avoiding interaction, which is ultimately set to its fixed point value of $u^{*}=2 \pi^{2} \epsilon[25,26,30]$. To calculate the scaling properties for $r \gg a$, it is sufficient to include only the first term $(k=1, l=m=0)$ from Eq. (10) in the non-loop propagators (as in the wedge computation by Cardy [25, 26], higher-order terms give corrections in higher powers of $a / r)$. The intermediate loop, however, can be of any size requiring the entire sum (albeit regularized by subtracting the result for $\Theta=0$ to remove an unrelated divergence). The loop correction is an integral over the whole space. The integration over angles $\phi^{\prime \prime}$ and $\psi^{\prime \prime}$ is trivial due to symmetry and yields $l(l+1)$ when summed over all spherical harmonic functions of degree $l$; in the integration over the radius $r^{\prime \prime}$ we seek a logarithmic contribution which is exponentiated in the end. Similar to Cardy's analysis, such a logarithm comes only from the region $r<r^{\prime \prime}<r^{\prime}$. There remains an integral over the polar angle $\theta^{\prime \prime}$, but this is cumbersome as we have to find the roots to the transcendental equation noted before. We shall not dwell further on the $\epsilon$-expansion for a general opening angle. Instead, we focus on the limit of a sharp cone as $\Theta \rightarrow 0$, where the leading singularity comes only from the $l=0$ channel of the loop Green's function. This is because a sharp cone couples only to the lowest spherical partial wave. A careful integral over $\theta^{\prime \prime}$ and summation over all $k$ 's gives the radial dependence of the Green's function as

$$
G_{1} \propto \frac{r^{\eta_{\mathrm{c} 0}}}{r^{\prime} \eta_{\mathrm{c} 0}+2}\left[1+\epsilon \ln \frac{r}{r^{\prime}}\left(\frac{1}{4 \pi} \Theta \ln \Theta+.16 \Theta\right)\right],
$$

where the term proportional to $\epsilon$ in the bracket is the one-loop correction to the Green's function. In $d=4-\epsilon$ dimensions, $\eta_{\mathrm{c} 0}$ is given by Eq. (9). Now we can exponentiate the radial logarithm to obtain the renormalized exponent,

$$
\eta_{\mathrm{c}}=\eta_{\mathrm{c} 0}+\left(\frac{\Theta \ln \Theta}{4 \pi}+.16 \Theta\right) \epsilon .
$$

Note that the loop-correction vanishes logarithmically with the angle, as there is no first order in $\epsilon$ contribution to $\eta$ in empty space. Repeating the same procedures for the cone-plate we find, as $\Theta \rightarrow 0$,

$$
\eta_{\text {cp }}=\eta_{\text {cp } 0}+\left(-\frac{1}{8}+\frac{\Theta \ln \Theta}{\pi}+.66 \Theta\right) \epsilon,
$$

where the exponent $\eta_{\mathrm{cp} 0}$ is given by Eq. (9). In this case, the loop-correction goes to $-\epsilon / 8$ for $\Theta=0$ due to the presence of the plate. Equations (13) and (14) suggest interpreting the logarithmic corrections as signatures of a power-law for $\Theta \rightarrow 0$. Expanding $\eta_{\mathrm{c} 0}$ and $\eta_{\mathrm{cp} 0}$ to the first order in $\epsilon$, we find another $\ln \Theta$, originating from the expansion in $4-\epsilon$ dimensions of the phantom polymer (as opposed to the perturbative terms from the one-loop computation). Summing both contributions, we obtain

$$
\begin{aligned}
\eta_{\mathrm{c}} & =\frac{\Theta}{\pi}\left(1-\frac{3}{4} \epsilon \ln \Theta-.06 \epsilon\right), \quad \text { and } \\
\eta_{\mathrm{cp}} & =1-\frac{\epsilon}{8}+\frac{4 \Theta}{\pi}\left(1-\frac{3}{4} \epsilon \ln \Theta-.86 \epsilon\right) .
\end{aligned}
$$

We can then recast the approach of the exponents to their limiting values as a power-law $\Theta^{p}$ with $p=1-3 \epsilon / 4$ in place of $p_{0}=d-3=1-\epsilon$ for the phantom polymer (Fig. 21). We may interpret this result as follows: self-avoidance swells the polymer, reducing its fractal dimension from 2 to $\nu^{-1}=2-\epsilon / 4$ to lowest order. The dimensionality of the intersection of such an object with the remnant line is $d-1-\nu^{-1}=1-3 \epsilon / 4=p$. We leave this observation as a conjecture for future studies.

From a practical point of view, the amplitude $\mathcal{A}=$ $\eta_{\mathrm{cp}}-\eta_{\mathrm{c}}$ is typically a number of order unity. Thus the force in Eq. (11) at room temperature is roughly around $0.1 \mathrm{pN}$ at $0.1 \mu \mathrm{m}$ separation; at the margins of possible measurements by current force apparatus. The force can be increased by attaching several polymers to the tip. For ideal (phantom) polymers the force is enhanced by $f$, the number of polymers, while interactions modify this conclusion. The interactions are of two kinds: self-interaction of a single arm (intra-arm) and interactions between two different arms (inter-arm). The former contribution is computed above to first order in $\epsilon$ and should be multiplied by $f$ for an $f$-arm polymer. The latter, however, gives rise to a new term multiplied by $f(f-1) / 2$, the number of pairs. Thus to first order in $\epsilon$, $\eta=f \eta_{0}+f \eta_{i}+\frac{f(f-1)}{2} \eta_{e}$ where $\eta_{0}$ denotes the exponent of a single phantom polymer, and $\eta_{i}$ and $\eta_{e}$ are those of intra-arm and inter-arm interactions respectively. In the absence of a cone, the set-up is similar to the widely studied case of star polymers [37]. We carried the corresponding $\epsilon$-expansion with cone and cone+plate. Here, we just quote the final result after appropriate exponentiations. We find (in the limit of sharp cones), a force amplitude

$$
\frac{\mathcal{A}(f)}{f}=1-\frac{\epsilon}{8}+\left[\frac{3}{\pi}-\left(.80+\frac{11}{12 \pi}(f-1)\right) \epsilon\right] \Theta^{1-3 \epsilon / 4} .
$$


Interactions amongst the polymers thus reduce the force amplitude per polymer.

Note that the same exponent $p=1-3 \epsilon / 4$ governs the approach to a finite limit in Eq.(16) as $\Theta \rightarrow 0$. Another experimental set-up is a cone-tip-attached polymer approaching another cone. The entropic force is of course much smaller in this case, and we have verified that it also vanishes with cone angle as $\Theta^{p}$, bolstering the conjecture that this is a universal exponent related to the needle geometry.

In summary, we propose that polymers exert an entropic force $\mathcal{A} k_{B} T / h$ on a cone tip, with a 'universal' amplitude $\mathcal{A}$ dependent on geometry, interactions, and number of polymers. We conjecture that the singular form of the amplitude on vanishing cone angle is described by a new exponent. There are many set-ups where a similar force law is expected on the basis of scaling at length scales shorter than an appropriate correlation length.

\section{ACKNOWLEDGMENTS}

This work was supported by the National Science Foundation under Grants No. DMR-08-03315 (MK, MFM), and PHY05-51164 (MK at KITP). Y.K. acknowledges the support of Israel Science Foundation grant 99/08. The authors acknowledge discussions with B. Duplantier and A. Grosberg.
[1] C. Bustamante, Z. Bryant, and S. B. Smith, Nature 421, 423 (2003).

[2] M. S. Kellermayer, Physiol. Meas. 26, R119 (2005).

[3] K. C. Neuman, T. Lionnet, and J.-F. Allemand, Annu. Rev. Mater. Res. 37, 33 (2007).

[4] A. A. Deniz, S. Mukhopadhyay, and E. A. Lenke, J. R. Soc. Interface 5, 15 (2005).

[5] K. C. Neuman and A. Nagy, Nature Methods 5, 491 (2008).

[6] T. E. Fisher, P. E. Marszalek, A. F. Oberhauser, M. Carrion-Vazquez, and J. M. Fernandez, J. Physiol. 520, 5 (1999).

[7] A. Kishino and T. Yanagida, Nature 334, 74 (1988).

[8] K. Neuman and S. Block, Rev. Sci. Instrum. 75, 2787 (2004).

[9] S. Hormeño and J. R. Arias-Gonzalez, Biol. Cell 98, 679 (2006).

[10] S. Gosse and V. Croquette, Biophys. J. 82, 3314 (2002).

[11] H. Kikuchi, N. Yokoyama, and T. Kajiyama, Chemistry Letters 26, 1107 (1997).

[12] C. Bustamante, C. Rivetti, and D. Keller, Curr. Opin. Struct. Biol. 7, 709 (1997).

[13] B. Drake et al., Science 243, 1586 (1989).

[14] P.-G. de Gennes, Scaling Concepts in Polymer Physics (Cornell University Press, Ithaca, New York, 1979).

[15] E. Eisenriegler, K. Kremer, and K. Binder, J. Chem. Phys. 77, 6296 (1982).

[16] B. Duplantier and H. Saleur, Phys. Rev. Lett. 57, 3179 (1986)

[17] P. Rowghanian and A. Y. Grosberg, J. Phys. Chem. B in print (2011).

[18] R. Bubis, Y. Kantor, and M. Kardar, Europhys. Lett.
88, 48001 (2009).

[19] E. Ben-Naim and P. L. Krapivsky, J. Phys. A: Math. Theor. 43, 495007 (2010).

[20] S. Redner, A Guide to First-Passage Processes (Cambridge University Press, New York, 1983).

[21] K. Binder, in Phase Transitions and Critical Phenomena, Vol. 8, edited by C. Domb and J. L. Lebowitz (Academic Press, London, 1983) p. 1.

[22] S. Caracciolo, M. S. Causo, and A. Pelissetto, Phys. Rev. E 57, R1215 (1998).

[23] N. Madras and G. Slade, The Self-Avoiding Walk (Birkhäuser, Boston, 1993).

[24] J. L. Cardy and S. Redner, J. Phys. A 17, L933 (1984).

[25] J. L. Cardy, Nucl. Phys. B 240, 514 (1984).

[26] J. L. Cardy, J. Phys. A: Math. Gen. 16, 3617 (1983).

[27] A. J. Gutmann and G. M. Torrie, J. Phys. A: Math. Gen. 17, 3539 (1984).

[28] M. N. Barber, A. J. Guttmann, K. M. Middlemiss, G. M. Torrie, and S. G. Whittington, J. Phys. A 11, 1833 (1978).

[29] K. De'Bell and T. Lookman, Rev. Mod. Phys. 65, 87 (1993).

[30] M. Slutsky, R. Zandi, Y. Kantor, and M. Kardar, Phys. Rev. Lett. 94, 198303 (2005).

[31] M. K. Kosmas, J. Phys. A 18, 539 (1985).

[32] J. F. Douglas and M. K. Kosmas, Macromolecules 22, 2412 (1989).

[33] P. Grassberger, J. Phys. A: Math. Gen. 38, 323 (2005).

[34] K. Suzuki, Bull. Chem. Soc. Japan 41, 538 (1968).

[35] Z. Alexandrowicz, J. Chem. Phys. 51, 561 (1969).

[36] M. F. Maghrebi, S. J. Rahi, T. Emig, N. Graham, R. L. Jaffe, and M. Kardar, PNAS 108, 6867 (2011).

[37] K. Ohno, Phys. Rev. A 40, 1524 (1989). 